\title{
Portable Health Monitoring Systems using Wearable
} Devices

\author{
Chang-Bae Noh' ${ }^{1}$ and Wonshik Na ${ }^{2 *}$ \\ 'Department of Electronic and Radio Engineering, Kyung-Hee University Yongin, \\ Republic of Korea; zzomenyduck@empas.com \\ 2Department of Computer Science, Namseoul University Cheonan, \\ Republic of Korea; winner@nsu.ac.kr
}

\begin{abstract}
Objectives: It is now the era of healthcare 3.0 and there is a growing interest in wellness care as a means to health management. Methods/Statistical Analysis: The system is not confined to specific locations or areas for it to sense and react to emergencies. The portable health monitoring system proposed in this study utilizes sensors embedded within the wearable devices to continuously measure the user's health and promptly alert relevant authorities and caretakers in case of an emergency. The portable health monitoring system proposed in this study needs to notify relevant authorities of emergency situations regardless of the user's location. Findings: In recent days even smartphones are being released that have embedded sensors much like wearable devices, however these are only meant to improve the convenience of the smartphones and should be differentiated from wearable devices that have been developed to fulfill specific purposes. The system proposed in this paper automatically checks the user's health in real time, vibrating the device as feedback in case of warning or danger. When within communication distance with a repeater via Bluetooth or NFC tags the system automatically forwards the collected data so that the users do not have to do so manually. Wearable devices are computers in the form of watches, bracelets, clothes, glasses, etc. that users can freely wear on their person. With the smartphone market reaching saturation the market for wearable devices with various add-on sensor functions is now attracting attention. The system also allows multiple users to share a repeater so that their information can be shared and ranked between the users, ultimately prompting users to be proactive in taking care of their health. Application/Improvements: This system can also be used as a form of big data by collecting the health information data of the middle-aged and the elderly and the real time feedback will allow a proactive management of the users' health.
\end{abstract}

Keywords: Health Monitoring, Sensor, SOS, Wearable Device, Wi-Fi

\section{Introduction}

The domestic smart device market has continued to experience an explosive growth until now. According to the '2014 Korean Society Indicator' by the National Statistical Office about $70 \%$ of the nation's population was found to be smartphone users, with 40,560,000 registered users in 2014. With the current smartphone market so saturated, wearable devices are now rising as the next-generation mobile device technology market that can be a new source of revenue. The 2014 February BCC Research report predicted that the wearable device market will see an average of $43 \%$ growth per annum from 5 billion USD in 2013 to 30.3 USD in 2018. Indeed, an increasing number of innovative wearable devices are being developed and released even today ${ }^{1}$. In light of such facts, this system introduces a wearable device that can be used with smart devices to frequently check and warn against collected data to create a smart wellness care service. This health monitoring system targets in the 30s 40s age bracket as well as families and allows users to compete against friends and families nearby as they take care of their health in a fun way ${ }^{2}$. Such wearable devices help to create an IoT where the internet is used to recognize situations in real life and provide

${ }^{*}$ Author for correspondence 
smart services and is used together with big data, sensors, etc. in a variety of ways to create a new market ${ }^{4}$ as shown in Figure 1.

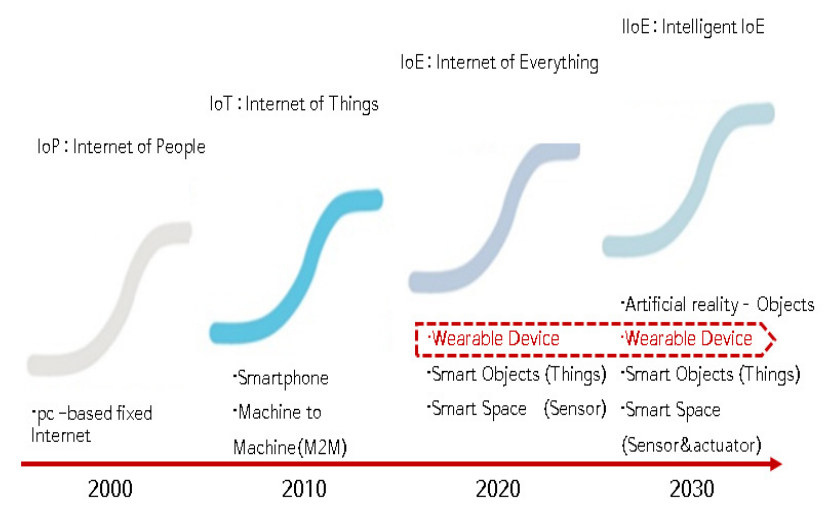

Figure 1. The intelligent IoE paradigm and a new IT strategy for future planning $\underline{3}$.

\section{Wearable Devices}

Wearable devices are computers in the form of watches, bracelets, clothes, glasses, etc. that users can freely wear on their person. With the smartphone market reaching saturation the market for wearable devices with various add-on sensor functions is now attracting attention ${ }^{5}$. In recent days even smartphones are being released that have embedded sensors much like wearable devices, however these are only meant to improve the convenience of the smartphones and should be differentiated from wearable devices that have been developed to fulfill specific purposes. The health information data that is automatically measured at all times will be accumulated in the smart device and will be forwarded automatically via Bluetooth if the repeater comes within communication distance. The data can also be forwarded manually via NFC. The received data is analyzed in the repeater and shown on the repeater screen. The portable health monitoring system proposed in this study utilizes a new technology based on healthcare device development that maximizes user convenience by automating the previously manual healthcare process. It is expected to have a positive effect on reducing the time and cost needed to develop devices that utilize the technology, thereby contributing to their increased competitiveness. In order to encourage consumers to purchase such devices it is essential to use the devices' power efficiently by only including necessary functions, instead of including too many functions in the development stage.
'Wearable' is originally a fashion term meaning 'it can be worn on the body'. Combined with technology, wearable here can be defined as IT devices that can be worn on the body ${ }^{6}$. Many commercialized devices focus on functionality and technology. However, what consumers seek are devices that combine functionality and technology while also satisfying their need to express their individuality and sense of fashion. Thus, there is a need to develop wearable devices that perform distinctive functions for a specific purpose as opposed to devices that act as a secondary device ${ }^{7}$ as shown in Figure 2.

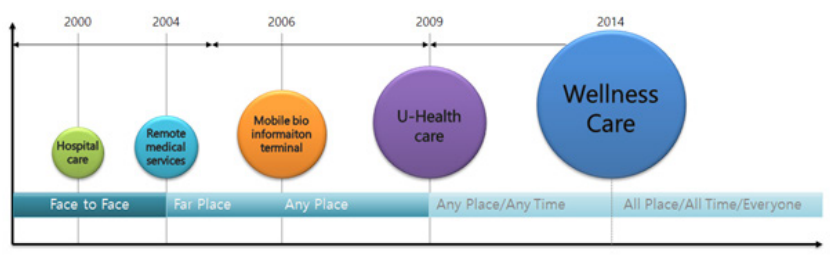

Figure 2. Developments in healthcare technology.

\section{Health Information Processing Technology}

It is now the era of healthcare 3.0 and there is a growing interest in wellness care as a means to health management. The aim of this study is to develop a smart device that checks the user's health state and provide real time feedback with warning and danger alerts thus prompting proactive health management, not just alone but with others as well and thereby allowing a fun social network healthcare management. Arduino sensors and exercise equipment (waist exercise) will be used to check and collect users' health information as well as their waist measurement, body temperature, pulse, exercise load, blood sugar, etc. The data can be stored in the smart devices $^{-}$as shown in Figure 3. When the user exercises the sensors in the handles check some of the user's health information. The results will not be immediately available and will only be accessible after a certain amount of exercise has been done. Furthermore, specific health data can be checked in real time and warning alerts via vibrations can be given when the value exceeds the previously set amount. The vibration will not automatically turn off after a given amount of time and instead will require the user to check the value and turn it off manually. The data will be automatically forwarded when the user comes within communication distance to a repeater, upon which the 
data will be analyzed and displayed to the user ${ }^{9}$ as shown in Figure 4. The system prompts users to manage their health in their everyday lives in a natural way. If needed, the system can also be used to forward the collected data to medical institutions or to compete with other users. The system provides a healthcare service that utilizes social networking services (video calls) unlike existing systems that only allow solitary healthcare management $t^{10}$.

Current temperature: $20 \mathrm{C}$

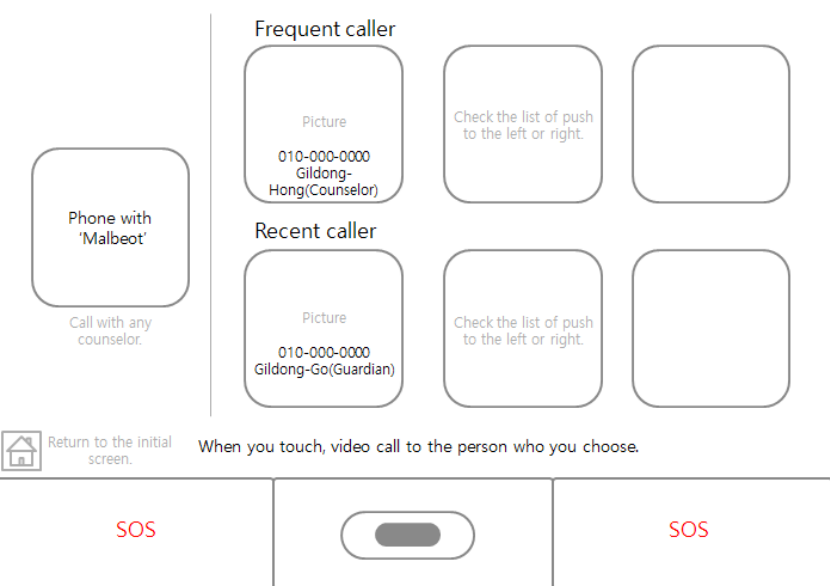

Figure 3. System design for the portable and wearable health monitoring device.
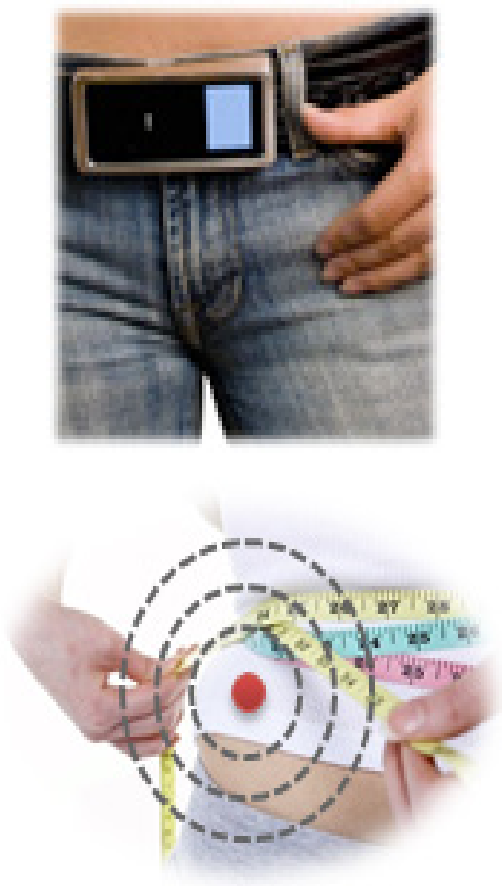

Figure 4. Smart device worn by the user and real time checking of data (Feedback).

\section{Proposed System}

The wearable device system proposed in this study utilizes previous research, a connection to a 365 homecare health service and a small Wireless Body Area Network (WBAN) between the devices to swiftly alert relevant authorities and guardians when problems arise with the user's health. The temperature sensors, pulse sensors and the GPS sensors embedded in the wearable device can sense fires and other emergency situations to use $4 \mathrm{G}$ or $3 \mathrm{G}$ to forward the user's status and location information via Wi-Fi or the Bluetooth on smartphones to the 365 homecare health service TAB as shown in Figure 5. The TAB forwards the information to relevant institutions and persons so that swift action can be taken. This portable health monitoring system can be made to look like an accessory in general as it does not require a large device since it does not process information in itself, nor a big display monitor as shown in Figure 6.

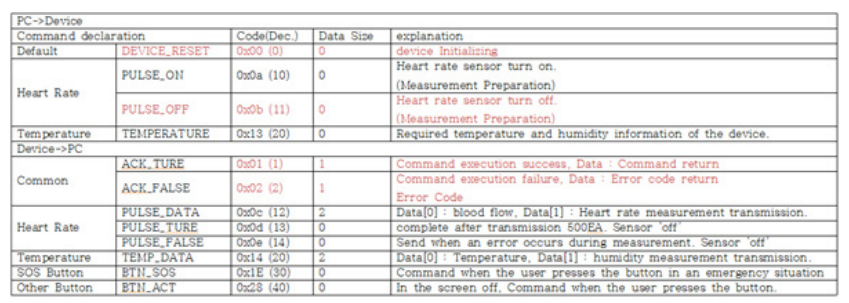

Figure 5. Command definition.

\begin{tabular}{|c|c|c|c|c|}
\hline $\begin{array}{l}\text { Accelerometer } \\
\text { yyroscope } \\
\text { Compess }\end{array}$ & Heart Rate & Ambient Light & $\begin{array}{l}\text { Altimeter } \\
\text { GPS }\end{array}$ & $\begin{array}{l}\text { Blood } \\
\text { Oxygen }\end{array}$ \\
\hline $\begin{array}{l}\text { Track Physical } \\
\text { Actinty }\end{array}$ & Cardio & Sleep qualiny & \begin{tabular}{|c} 
Physical Activity \\
Tradx
\end{tabular} & Respiratory check \\
\hline Fall Detection & Fitness Level & UV Expossure & Fitness & Fitness Level \\
\hline Step canter & Gym & & Calonies & Anesthetic Moniter \\
\hline Calories Bum & $\begin{array}{l}\text { Heart Problem } \\
\text { Waming }\end{array}$ & & & $\begin{array}{l}\text { Heart Problem } \\
\text { Waming }\end{array}$ \\
\hline Sleep Quality & & & & \\
\hline
\end{tabular}

Figure 6. Sensor technology-based health care elements.

\subsection{User Health Monitoring}

When the user wears the wearable device for the first time they will set 364 homecare health service TAB information as well as the standard (numerical value) to which danger levels will be calculated. Then, the user's pulse and surrounding temperature information will be constantly checked through the sensors $\frac{11,12}{2}$. In this state the data will not be saved and the Wi-Fi will not be connected either to conserve battery. When the danger level is reached 
the emergency process outlined in 4.2 is triggered. Due to the fact that the data is not always saved, there is no need for a large storage space and the limited battery can be effectively used as in Figure 7. The definitions for the communication packet structure for the portable health monitoring system are as such in Figure 8.

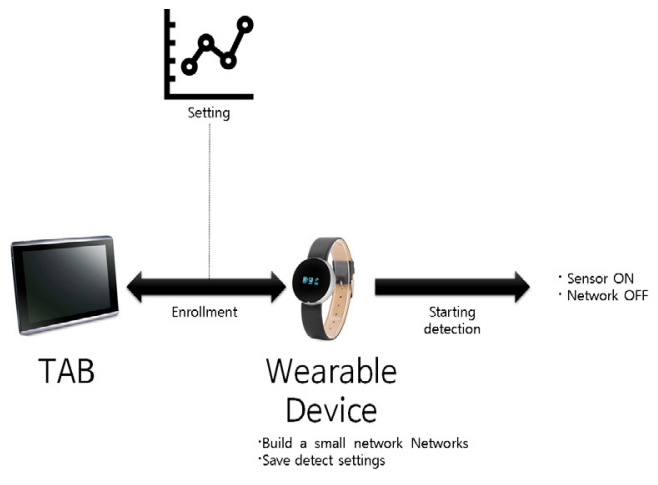

Figure 7. Summary of the health monitoring system.

\begin{tabular}{|c|c|c|c|c|c|c|}
\hline STX & 10 & CMO & DATA SIZE & DATA & CHECK SUM & ETX \\
\hline 1 Bute & 1 Byte & 1 8yte & 1 Byte & $n$ Byte & 1 Byte & $18 \times 8$ \\
\hline $\begin{array}{l}\left.P^{\prime}(0 \times 3 E)\right\} \\
\langle(0 \times 3 C)\end{array}$ & $\begin{array}{c}P C=0 \times 00 \\
B O A B O=0 \times 01\end{array}$ & PUISE ON & $0 \times 00 \sim n$ & D n Byte & $\begin{array}{c}\text { STX+1D+CMD+ } \\
\text { DS+DATA \% } 256= \\
0 \times 00 \sim 0 \times A f\end{array}$ & $\because 0 \times 38$ \\
\hline
\end{tabular}

Figure 8. Communication packet structure.

\subsection{Emergency Process}

The device attempts to connect to the Wi-Fi. If the connection fails the device will forward the data to the smartphone using the user's smartphone Bluetooth function. The smartphone will receive the data using $3 \mathrm{G}$ and $4 \mathrm{G}$. When the preparation is done to forward the data, the device will location information from the GPS sensor and the detected danger (fire detection via the temperature sensors, emergency detection via the heart rate measurement) to the homecare health service TAB installed in the user's home. The TAB will forward and record an emergency SOS message to relevant authorities and guardians.

\subsection{SOS Service}

While the sensors can automatically carry out the notification process in case of emergency, there may be cases where the sensors do not work properly or there are exceptional emergency situations when the user can start the same emergency process with a push of a button. When the emergency process is started manually, only the GPS information will be forwarded to the TAB as shown in Figure 9.

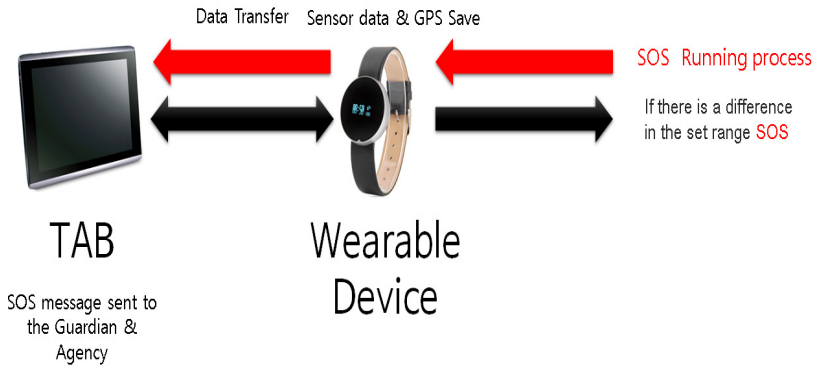

Figure 9. SOS process.

\section{Conclusion}

The portable health monitoring system proposed in this study needs to notify relevant authorities of emergency situations regardless of the user's location. There should be continued research on how to determine the user's location as well as to reduce the amount of time needed for the emergency processing and sending the SOS message. Aside from the temperature and pulse sensors there should be various additional methods that can measure the user's physical information and the external environment and the scope of the care system should be gradually increased to include not only accessories but also clothing and more. Such health management will have the effect of preventing diseases and cutting down on medical costs as well as maximizing users' convenience, while also increasing the competitiveness of similar products by reducing the amount of time needed to develop devices utilizing the same technology as well as their costs. In addition, this system can also be used as a form of big data by collecting the health information data of the middleaged and the elderly and the real time feedback will allow a proactive management of the users' health.

\section{Acknowledgment}

Funding for this paper was provided by Namseoul University.

\section{References}

1. Lee SH, Jeong YS. Information authentication selection scheme of IoT devices using conditional probability. Indian Journal of Science and Technology. 2016 Jun; 9(24):1-7.

2. Tompkins W. Biomedical digital signal processing: C-language examples and laboratory experiments for the IBM PC. Upper Saddle River, NJ, USA: Prentice-Hall Inc; 1993. 
3. Son MS. Wearable system for u-health service support. Electronic Communication Trend Analysis Book; 2006 Sep.

4. Han J, Seo L. Study on the context awareness that the order search system in a distributed computing environment. Indian Journal of Science and Technology. 2015 Jul; 8(13):1-8.

5. Mel HX, Baker D. Cryptography decrypted. Addison Wesley; 2001.

6. Prakash M, Gowshika U, Ravichandran T. A smart device integrated with an Android for alerting a person's health condition: Internet of Things. Indian Journal of Science and Technology. 2016 Feb; 9(6):1-6.

7. Lee SH, Kim M, Chung H, Yang S. Designing of efficient technique blocking abnormal packets through correlation analysis in the healthcare environment. Indian Journal of Science and Technology. 2016 Jun; 9(24):1-5.
8. Schwab EC, Nusbaum HC, Carpenter GA. Pattern recognition by humans and machines. Academic Press; 1986 Jan.

9. MoMoreno A, Garbay C. Software agents in healthcare. Artificial Intelligence in Medicine. 2003; 27:229-32.

10. Weiss G. Multi-agent systems. A modern approach to distributed artificial intelligence. MIT Press; 2000 Sep.

11. Exercise for depression. 2008. Available from: http:// onlinelibrary.wiley.com/doi/10.1002/14651858.CD004366. pub3/abstract

12. Moon HY. Macrophage migration inhibitory factor mediates the antidepressant actions of voluntary exercise. Proceeding of National Academy Science, U.S.A. 2012 Aug; 109(32):13094-9. 